\title{
Television analyzer of aerosol particles images
}

\author{
Vladimir Semenov ${ }^{1, *}$, Yurij Hanzhonkov ${ }^{1}$, Yurij Astsaturov ${ }^{2}$, and Victoria Shvets ${ }^{1}$ \\ ${ }^{1}$ Don State Technical University, Department of Radio-electronic and electro-technical systems, \\ 147 Shevchenko st., 346500 Shakhty, Russia \\ ${ }^{2}$ Don State Technical University, Department of Technical operation of cars, 147 Shevchenko st., \\ 346500 Shakhty, Russia
}

\begin{abstract}
In the paper an aerosol image analyzer is described, which allows to obtain in three projections the main geometric and optical parameters of the aerosol particles. It is necessary for the calibration of simple optical-electronic sensors. We describe a recognition algorithm that allows real-time obtaining of data on the shape, size, and color of studying aerosols.
\end{abstract}

\section{Introduction}

Simple optical aerosol particles detector requires calibration for each types of dust or aerosol suspension. The calibration is depending on size and shape of aerosol particles.

Among various methods for determining the size and shape of suspended particles, television methods are the most informative $[1,2]$. Usually, one image of each particle is recorded, corresponding to its projection on a plane perpendicular to the optical axis of the light beam.

Significantly increase the information content of measurements using this method allows obtaining two or more (for more complex shapes) particle images. It leads to increasing cost and complexity of particles measurement system [3].

In this paper we propose a method of obtaining 3 projections of aerosol particles images on co-perpendicular planes simultaneously.

\section{The analyzer description}

The functional diagram of proposed analyzer is shown in the Figure 1 [4]. Dynamic injection of aerosols into the area of control is carried out by a pumping cell with a channel width of $1.5 \mathrm{~mm}$ and a vacuum pump. The flow speed is selected by PC program so that no more than 1 particle (50 particles per second) gets into each frame of the video signal. Control of flow speed realised by dust particle photoelectric counter (PEC), the NI PCI$6024 \mathrm{I} / \mathrm{O}$ card and personal computer (PC) which are inserted into the analyzer functional diagram. The control program conducts amplitude and frequency analysis of the input signal from the PEC for preliminary detection of the particle sizes. The control signal

\footnotetext{
*Corresponding author: semenov@sssu.ru
} 
generated by the program comes from the PC and the NI PCI-6024 I/O board for controlling the flow speed (vacuum pump) and focusing the video analyzer camera lens.

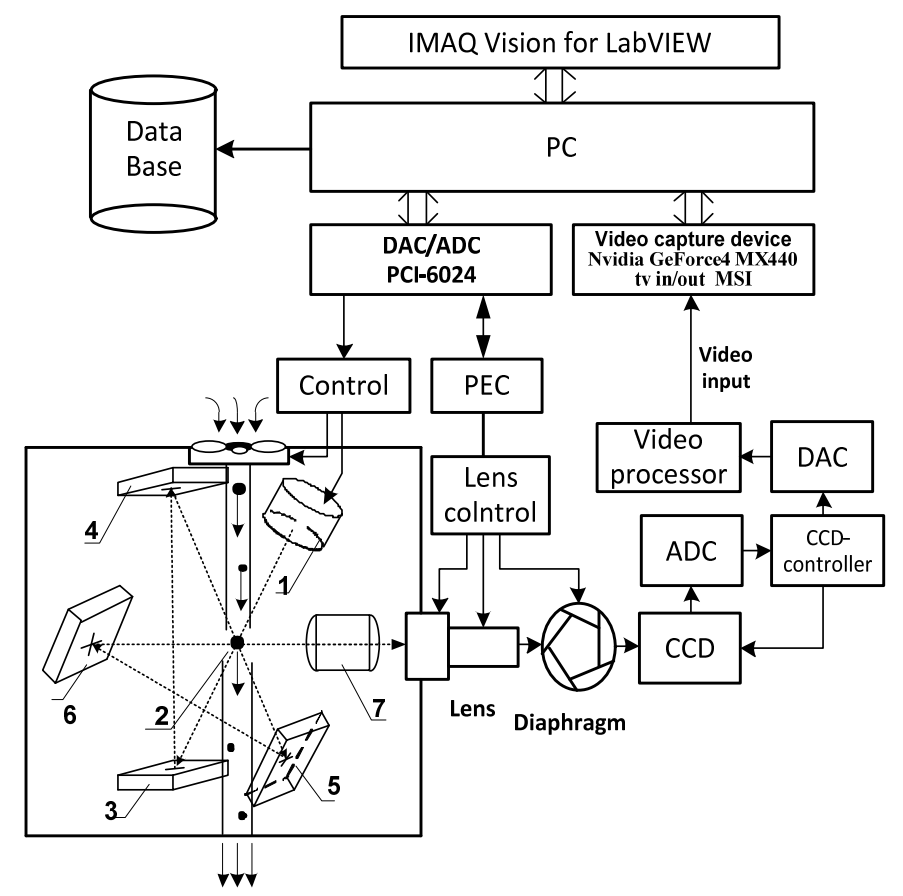

Fig. 1. Functional diagram of proposed analyzer.

In the Figure 1:

1 - LASER source; 2 - aerosol flow; 3, 4, 5, 6- mirrors; 7 - micro-lens; Control - the control device for LASER and pump; PEC - photoelectrical counter; Lens control - the control module for focus, zoom, diaphragm and exposition; CCD - charge-coupled device matrix; ADC, DAC, Video processor - video camera system, Data base - the table of particles parameters.

In the Figure 2 the PEC block diagram is shown. The particles of dust in the measuring chamber dissipate the laser beam. This diffused light goes to photomultipier tube where the electrical signals are generated. This electrical signals pass through I/O board (NI PCI-602) to PC. The measurement results are displayed on PC monitor. PC controls laser and pump by program controlled electrical switches within I/O board.

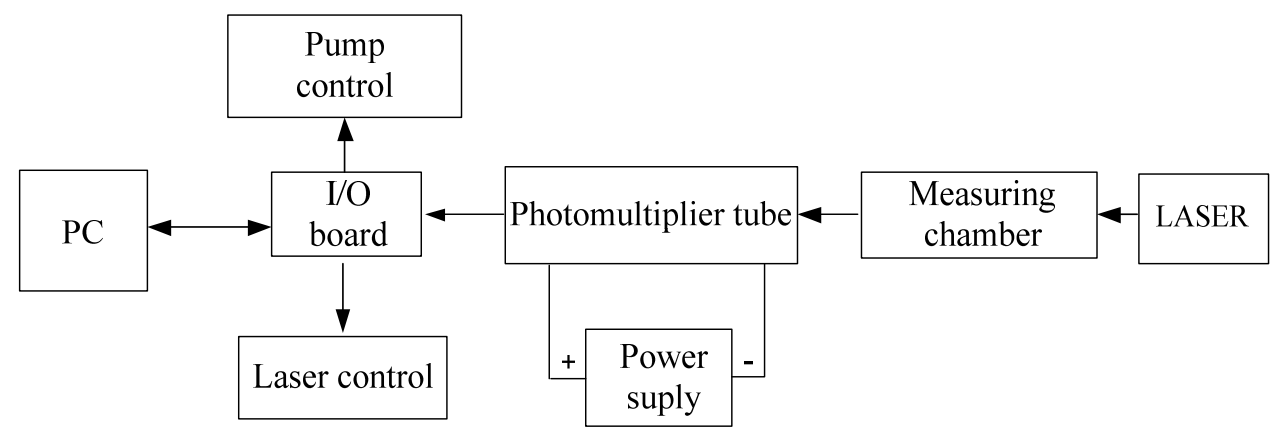

Fig. 2. Functional diagram of proposed analyzer. 
The experimental equipment consists in two pieces. First one is television analyzer and the second part is PEC. Appearance of experimental equipment and work screen of software is shown in Figure 3.

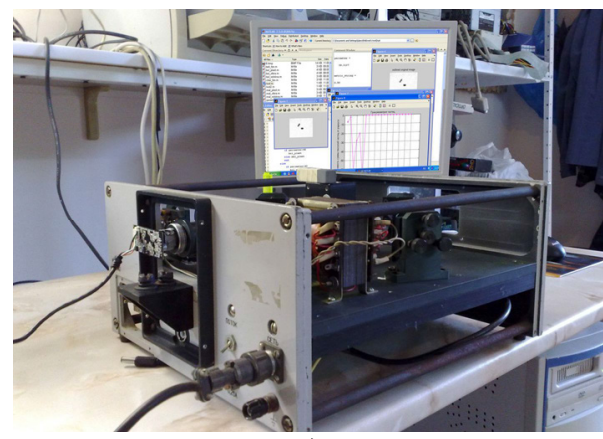

a)

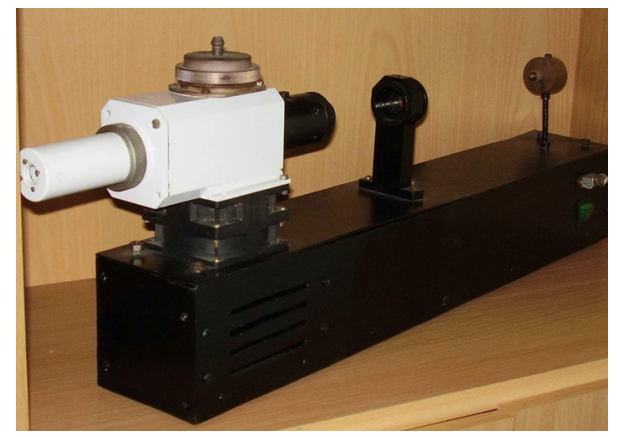

b)

Fig. 3. Experimental equipment ( $a-$ television analyzer, $b$ - PEC).

\section{Results}

The images of dust particles are shown in Figure 4. We have to note that low measurement level by size is determinate by CCD and optic system resolution. It is proximally 1 micrometer in any dimensions. And the measuring chamber volume (aerosol concentration) is $10^{5} \mathrm{~cm}^{3}$.

Measured aerosol particles may have different shapes [5]:

1. Isometric particles. They have almost same dimensions for three axes. It can be spheres, regular polyhedral or near that. This type is most studied.

2. Plates - the particles that have long size in two axes and one short dimension. It can be petals, flakes, scales, discs etc.

3. Fibers - the particles that have long size in one axes and short in other two. It can be knits, prisms, needles, mineral fibers etc.

Applied methods of digital image processing are depended on particle shapes.

The special algorithm of digital image processing is developed. The first stage of the algorithm is to classify shape of particle: isometric, plate or fiber. On the second stage every classified particle is groped by size more or less than $30 \mathrm{um}$. For each group the next operations are performing:

1. Image displaying.

2. Contrasting and smoothing.

3. Filtering (median filtering, denoising).

4. Binarization. Edge detection.

5. Morphological analysis.

6. Counting.

7. Size spreading histogram building.

8. Storing data in data base.

The control program and processing algorithms was performed in LabVeiw [6]. The obtained data about size, shape and quantity of particles (about 60 parameters of particle) are stored in Excel table. These data are used for modeling and design optical-electric sensors for particular type of aerosol and dust particles. Modeling algorithms are based on Mie solution to Maxwell's equations (Mie scattering) and its variations for different shapes particles. 


\section{Conclusion}

Proposed obtaining of particles images method allows avoiding problems with synchronization of three axes projection cameras. In proposed method we use only one camera and obtain all projections at same time. It allows to determinate the aerosol particle shapes and to classify them for further electro-optical sensors calibration.

\section{References}

1. F. M. Shofner, G. Kreikebaum, and Jr A. C. Miller, U.S. Patent No. 4473296, 25.09.1984. (1984)

2. D. S. Covert, J. Heintzenberg, and H.-C. Hansson, Aerosol science and technology 12, 2, 446 (1990)

3. C. Bohern, D. Huffman, Absorption and scattering of light by small particles (John Wiley \& Sons, Weinheim, 1983)

4. V. Semenov, RUS Patent № 2436067, 22.10.2010 (2010)

5. P. Reist, Introduction to aerosol science (MacMillan, New York, 1984)

6. K. Thomas, Image processing with LabView and IMAQ Vision (Prentice Hall PTR, New Jersey, 2003) 